\title{
Post-mating Refractoriness in Drosophila melanogaster Depends Upon Ecdysis
} Triggering Hormone Signaling

Matthew R. Meiselman ${ }^{1,2,3}$, Anindya Ganguly ${ }^{2}$, Anupama Dahanukar ${ }^{1,2}$, Michael E. Adams ${ }^{1,2,3}$

${ }^{1}$ Graduate Program in Cell, Molecular and Developmental Biology, University of California, Riverside, CA, 92521, USA

2 Department of Molecular, Cell, and Systems Biology, University of California, Riverside, CA, 92521, USA

${ }^{3}$ Department of Entomology, University of California, Riverside, CA, 92521, USA

\section{Abstract}

An individual's decision to engage in courtship depends on external cues from potential mates and internal cues related to maturation, health, and experience. Hormones allow such information to be conveyed to distal tissues in a coordinated fashion. Here, we show EcdysisTriggering Hormone $(\mathrm{ETH})$ is a regulator of male courtship in Drosophila melanogaster, and critical for mate choice and courtship inhibition after the completion of copulation. Preventing ETH release increases male-male courtship and decreases post-copulation courtship inhibition (PCCI). Such aberrant male courtship behavior in ETH-deficient males appears to be the consequence of inabilityto integrate pheromone cues into decision making. Silencing of ETH receptor (ETHR) in GR32A-expressing neurons leads to reduced ligand sensitivity and elevated male-male courtship. We find OR67D is critical for suppression of courtship after mating, and ETHR silencing in OR67D-expressing neurons, and GR32A-expressing neurons to a lesser degree, elevates post-copulation courtship. Finally, ETHR silencing in the corpus allatum 
increases post-copulation courtship; treatment of with juvenile hormone analog partially restores normal post-mating behavior. ETH, a stress-sensitive reproductive hormone, appears to coordinate multiple sensory modalities to guide Drosophila male courtship behaviors, especially after mating.

\section{Introduction}

Modulation of sensory perception is critical for prioritization of appropriate behaviors under varying physiological conditions. For example, in the fruit fly Drosophila melanogaster, mating is costly for both sexes (Chapman et al., 1995; Markow et al., 1978), and the decision to engage in mating behaviors must be weighed with internal state and probability of success (Ejima et al., 2007; Zhang et al., 2019). Drosophila males weigh these decisions with a network of fruitlesspositive neurons (Demir and Dickson, 2005). Reproductive decision-making and consequent behavioral output in male flies is dictated by the activity of P1 neurons (Kimura et al., 2008; Pan et al., 2012). These neurons integrate olfactory, gustatory, visual, and hormonal queues to determine arousal and the ultimate decision to initiate mating (Clowney et al., 2015; Ribeiro et al., 2018; Wu et al., 2019). In addition to direct modulation of the arousal hub, it appears that primary sensory neurons that are necessary for mate perception are also modulated by hormonal state (Lin et al., 2016). This layered control allows the organisms to integrate a variety of factors into their ultimate decision of whether or not to mate.

We recently reported that Ecdysis Triggering Hormone (ETH) persists into the adult stage and acts as a stress hormone in the female, dramatically altering reproductive state (Meiselman et al., 2017; Meiselman et al., 2018). Here, we show that blocking ETH release or elimination of Inka cells, the sole source of ETH, relieves post-copulation courtship inhibition (PCCl) and increases male courtship toward conspecific females and males. Similarly, ETH receptor (ETHR) 
knock down in aversive pheromone-sensing neurons that express GR32A and OR67D promotes post-copulation and male-male courtship, suggesting that ETH modulates courtship by affecting pheromone sensitivity. While juvenile hormone $(\mathrm{JH})$ levels are partially responsible for postcopulation courtship inhibition, but treatment with the juvenile hormone analog methoprene (JHA) partially rescues the behavior. We conclude that ETH, a stress-sensitive reproductive hormone, is a potent modulator of courtship, and necessary PCCl.

\section{Results}

\section{ETH Deficiency Disinhibits Male Courtship Behavior}

Normal levels of male courtship behavior depend on JH (Wijesekera et al., 2016), we examined whether ETH-deficient males were impaired in mating efficacy. Surprisingly, we found that ETH deficiency, caused either by expressing the pro-apoptotic protein reaper in the Inka cells during the adult-stage (ETH-Gal4;Tubulin-Ga/80ts $>$ UAS-Reaper)(White et al., 1996), or by adult-specific prevention of ETH release by overexpression of the temperature-sensitive version of the dynamin mutant, shibire ${ }^{t s}\left(E T H-G a l 4>U A S-\right.$ shibire $\left.^{t s}\right)($ Gonzalez-Bellido et al., 2009), causes increased male-female copulation success in a ten-minute window (Figure 1A-B). Injection of ETH peptide to wild-type Canton-S males just before being placed with a female dramatically attenuates courtship (Figure 1C). Additionally, both manipulations caused a dramatic increase in courtship toward conspecific males (Figure 1D-E). Most strikingly, we found that ETH-deficient male courtship toward females did not terminate after successful mating, as was observed in control flies (Figure 1G, S1A-B, Supplementary Video 1). In fact, postcopulation courtship in Inka cell-killed males was not significantly different from pre-copulation courtship, though courtship declines dramatically after mating in wild-type, Canton-S flies and 
controls (Figure 1F). This was unlikely to be due to incomplete mating, as copulation duration for ETH-deficient males was significantly longer than controls (Figure S1C-D). We also found that silencing the ecdysone receptor in the Inka cells (ETH-Gal4;Tub-Gal80ts>UAS-EcR-RNAi), which reduces ETH expression (Cho et al., 2014), also stimulates post-copulation courtship (Figure S1E).

Male flies typically reduce courtship overtures after mating (Jallon, 1984). This reduction depends upon internal cues from copulation reporting neurons which suppress courtshippromoting NPF neurons (Zhang et al., 2016, 2019), and sensing of external cues which signal female refractoriness (Gillott, 2003; Laturney and Billeter, 2016; Vandermeer et al., 1986). Males deposit pheromones including cis-Vaccenyl Acetate (cVA) and 7-tricosene (7T) on females during copulation, which discourage males from courting during future encounters (Bontonou and Wicker-Thomas, 2014). ETH-deficient males may therefore either be impaired as "senders" (inability to deposit anti-aphrodisiacs) or "receivers" (inability to perceive or integrate information from anti-aphrodisiacs). To clarify, we performed a "Mate-and-Switch" experiment, mating ETHdeficient and wild-type males simultaneously, and swapping their former mates after completion (Figure 2A). We found Canton-S (control) males court females formerly mated to ETH-deficient males at low levels, whereas ETH-deficient males courted mated females at high levels (Figure 2B). These findings suggest that ETH modulates internal state to tune courtship. Taken together, ETH levels appear to be critical for maintaining normal courtship behaviors after mating.

\section{ETHR-silencing impairs GR32A Neuron Function and Elevates Male-Male Courtship}

Males and mated females rely upon pheromones to communicate toward other males that they are an unsuitable mate (Rings and Goodwin, 2019). We recently showed that suppression 
of male-male courtship depends upon ETH signaling in antennal lobe interneurons and that $\mathrm{JH}$ levels and ETH-JH signaling does not impact male-male courtship (Deshpande et al., 2019). ETH-deficient males court conspecific males with more than twice the intensity reported from interneuron-specific ETHR-silencing, we manipulated ETHR expression to determine the extent of ETH modulation of other courtship-related neurons. First, we silenced ETHR pan-neuronally, and observed a significant elevation of post-copulation courtship behavior (Figure 2C). ETHRtrojan-Gal4 expression is fairly restricted in the brain, labeling Kenyon cells, peptidergic neurons, and olfactory neurons (Lee and Adams, 2021). We identified fruitless-positive, courtshipregulating olfactory neurons innervating VA1Im, VL2A, and DA1 glomeruli, which are postsynaptic targets of OR47B, IR84A, and OR67D, respectively (Figure 3A). ETHR-Gal4 also labeled taste neurons in the labellum, including those innervating S-type sensilla, which house deterrent neurons that sense bitter compounds and pheromones that inhibit courtship. ETHRGal4 labeled taste neurons in male tarsi but, interestingly, not in female tarsi (Figure 3B-C). Also, ETHR and GR32A (ETHR-trojan-Gal4;UAS-mCD8>GR32A-lexA;AOPmCherry) expression overlaps in the labellum, but not in the tarsi (Figure S2A-B). GR32A, OR47B and OR67D all sense male-derived volatiles (Kurtovic et al., 2007; van Naters and Carlson, 2007; Wang et al., 2011). As ETH-deficient males court other males intensely, we assessed the requirement of ETHR in each of these classes of neurons for inhibition of male-male courtship (Figure 3D). Males with ETHR knocked down in GR32A neurons, but not in OR47B or OR67D neurons, significantly increased courtship toward conspecific males, suggesting that ETH may influence GR32A neuron sensitivity.

The overlap in labellar but not tarsal expression of GR32A-LexA and ETHR-Gal4 suggests ETH may target pheromone-sensing neurons in the labellum. GR32A-positive neurons in the 
labellum are activated by 7T as well as a variety of other bitter tastants (Lacaille et al., 2007; Moon et al., 2009). We tested response to denatonium in ETHR-silenced (GR32A-Gal4>ETHR$R N A i$ ) and control males, and found fewer action potentials with a longer latency to response over a range of concentrations (Figure 3E-F). Thus, ETHR deficiency may result in diminished sensitivity to ligands in primary sensory neurons, likely including pheromones.

ETHR Silencing in OR67D and GR32A-expressing neurons relieves post-copulatory courtship inhibition (PCCI) status to future suitors (Laturney and Billeter, 2016). These pheromones are sensed by OR67D and GR32A neurons, whose activity inhibits courtship. We investigated whether these pathways are important for inhibition of courtship immediately after mating. We assessed whether OR67D-

I- and GR33A'-- (a bitter receptor co-expressed with Gr32a and involved in sensing pheromones

that inhibit male-male courtship) knock-out mutants were impaired in post-copulation refractoriness (Figure 4A). Only OR67D ${ }^{-/-}$showed elevated courtship after copulation, suggesting that this pathway is indeed a critical component of PCCI. We silenced ETHR in GR32A and OR67D-expressing neurons and, surprisingly, found that ETHR-silencing in both subsets relieves $\mathrm{PCCl}$ (Figure 4B). These results suggest that ETH signaling modulates pheromone perception to tune male courtship both before and after mating. females, ETH-injected flies reduce courtship toward virgin females significantly, which do not 
courtship command neurons, preventing courtship (Zhang et al., 2021). We therefore sought to determine if $\mathrm{JH}$ plays a role in the disinhibition of post-copulation courtship observed in ETHdeficient males. We silenced the ETH receptor (ETHR) specifically in the CA, which reduces total JH levels (JHAMT-Gal4<UAS-ETHR-RNAi) (Meiselman et al., 2017). We observed a significant elevation in post-copulation courtship, suggesting $\mathrm{JH}$ may also play a role in postcopulation courtship (Figure 4C). We therefore sought to determine whether treatment with the JH analog, methoprene, could restore normal post-mating refractoriness (Figure 4D). Methoprene treatment of ETH-deficient males significantly decreased post-copulation courtship, and restored the normal post-mating decline of courtship, but did not restore courtship to wildtype levels (Figure 4D). JH appears to be critical for post-copulation courtship inhibition, but insufficient to completely account for elevated post-copulation courtship in ETH-deficient males. We therefore propose that ETH inhibits courtship by promoting $\mathrm{JH}$ synthesis and increasing sensitivity to courtship-inhibitory pheromones (Figure 4E).

\section{Discussion}

We show here that the peptide hormone ETH is necessary for courtship discrimination and for post-copulation courtship inhibition. We found that ETH-deficient flies show elevated courtship intensity toward same and opposite sex conspecifics, and, strikingly, ETH-deficient males do not terminate courtship after successful copulation. Furthermore, we found that ETHdeficient males are impaired in pheromone sensing or processing, and injection of ETH attenuated courtship intensity toward females. ETHR in GR32A-expressing neurons is necessary for normal responses to ligands and prevents abnormal courtship toward conspecific males. Post-copulation courtship is inhibited by OR67D, and ETHR-silencing in OR67D and 
GR32A-expressing neurons causes disinhibition of post-copulation courtship. Silencing ETHR in the CA also stimulates post-copulation courtship, and treatment of JHA to ETH-deficient males restores inhibition of courtship after mating.

Our data support a model that normal courtship depends upon ETH levels in the hemolymph. At low levels of ETH or with specific inactivation of ETHR, males are less responsive to courtship inhibitory pheromones, both at the level of the primary sensory neuron response and in behavior. JH inhibits courtship in early adulthood (Zhang et al., 2021), and ETH levels are tightly linked with $\mathrm{JH}$. It is therefore likely that ETH targets multiple, semi-redundant signaling systems (GR32A, OR67D, CA) to rapidly or dramatically change courtship levels in response to stimuli. Indeed, ETH is responsible for regulation of reproduction in response to stress, and downstream of ecdysone, a gonadotropic hormone that changes in titer in response to courtship, and can either stimulate or inhibit courtship depending on timing (Ishimoto et al., 2009).

\section{ETH Signaling in Primary Sensory Neurons}

ETH modulates a variety of courtship targets and appears to have a major influence over the drive to mate. Our previous report showed ETHR expression in antennal lobe interneurons is important for mate discrimination (Deshpande et al., 2019). Elimination of ETHR in antennal lobe interneurons caused disinhibition of male-male courtship, presumably through cVA desensitization (Liang et al., 2013; Liu et al., 2011). We show here that knockdown of ETHR in OR67D neurons regulates post-copulation courtship, which is also likely to be cVA-dependent. ETHR silencing in each disinhibits normal courtship, suggesting specific role for ETH in males. ETHR is a Gq-coupled GPCR, and stimulates calcium release from intracellular stores upon activation (Kim et al., 2006). During the ecdysis sequence, peptidergic ensembles of ETHR 
neurons mobilize calcium and release peptide signaling molecules that activate centrally patterned behaviors. In this study, excitability of ETHR-expressing chemosensory neurons may be under modulatory influences of circulating $\mathrm{ETH}$, becoming more or less sensitive to pheromone ligands as hormone levels fluctuate, as we observed in GR32A labellar neurons. It is currently unclear over what time frame such hormonal modulation in this context may occur, though during ecdysis, the correspondence between ETH release and behavioral outcomes is a matter of minutes (Kim et al., 2006; Park et al., 2003).

\section{ETH-JH Signaling Supports Post-Copulation Courtship Inhibition}

We recently demonstrated that ETH or ETHR deficiency decreases whole body juvenile hormone levels in both males and females (Meiselman et al 2017). In this work, we show that elimination of ETH or ETHR in the CA elevates courtship after mating. JH has been linked to male reproductive maturation and $\mathrm{JH}$ receptors in OR47B neurons promote their receptivity to courtship stimulatory pheromones (Lin et al., 2016). A recent report showed treating mature males with $\mathrm{JH}$ suppresses courtship and inhibits calcium activity in courtship-promoting NPF, doublesex-positive pCd neurons, and dopaminergic neurons (Zhang et al., 2021). JH levels do not influence male-male courtship (Deshpande et al., 2019), and post-copulation courtship does not seem ethologically beneficial at any life stage, arguing that this role for juvenile hormone is independent of homeostasis or development. JH targets Kenyon cells to mature and enhance learning memory (Lee et al., 2017; Leinwand and Scott, 2021), and drastically reduced JH levels could prevent flies from forming or accessing a "memory" of a recent, successful mating. Paired with sensory deficit, this may account for total elimination of post-copulation refractoriness.

\section{Conclusion}

Here, we established a relationship between ETH and courtship, wherein ETH stimulates 
activity in a variety of courtship-inhibiting targets (GR32A-expressing neurons, OR67Dexpressing neurons and the CA). We did not investigate how ETH levels may change innately and, in turn, manipulate male courtship. A drop in ETH levels in response to stress would by extension elevate courtship toward conspecifics, though in many other species stress has an inhibitory effect on male libido (Chenoweth, 1981; Lenzi et al., 2003; Marai et al., 2002).

We find the influence of ETH and $\mathrm{JH}$ in post-copulation courtship particularly intriguing, as the post-mating change in behavioral state has received less attention than pre-mating courtship. Further investigation is needed to determine how the CRN circuit, which attenuates activity of $\mathrm{P} 1$ neurons to induce mating satiety, integrates hormonal changes. After mating, females receive sex peptide, which is released slowly from sperm tails and causes infradian elevation of JH (Liu and Kubli, 2003; Moshitzky et al., 1996; Sugime et al., 2017), allowing females to accelerate oogenesis and replenish egg stores. The male must also replace accessory gland proteins after mating, which depend upon $\mathrm{JH}$ for their synthesis (Yamamoto et al., 1988). As males lack circulating sex peptide, this introduces the intriguing possibility that flies employ ETH, an innate allatotropin, to raise $\mathrm{JH}$ levels after copulation and inhibit post-copulation courtship. Indeed, in the closely related male carribean fruit fly Anastrepha suspensa, JH levels increase after mating (Teal et al., 2000).

We demonstrate here that courtship behavior is critically dependent on ETH signaling, and suggest multiple targets for this dependency. This investigation permits further investigation on how the now well-established neural circuitry of male courtship integrates endocrine state, particularly after mating.

\section{Acknowledgements}


231 We thank Roscoe Huo, Michael Chitgian, Lola Alade, Michelle Gyulnazaryan and Raymond Tan-

232 Tran for scoring courtship videos, Yuta Mabuchi for comments on the manuscript, Benjamin 233 White and Naoki Yamanaka for fly strains; Anandasankar Ray for fly lines and helpful advice; 234 and the Bloomington Stock Center (NIH P40OD018537) for reagents.

\section{Author Contributions}

237 M.M. and M.A. conceived the project and designed all the experiments. M.M. carried out all the experiments with undergraduate students mentioned in acknowledgements, with the exception of the electrophysiological recordings, which were designed, carried out, and analyzed by A.G. and A.D. M.M. and M.A. wrote the paper with feedback from all authors.

\section{Materials and Methods}

\section{Fly Strains}

under a 12:12 hr light:dark regimen. Inka cell-ablated flies were raised at the Gal80 ${ }^{\text {ts }}$ permissive temperature $\left(18^{\circ} \mathrm{C}\right)$. Following eclosion, they were moved to the nonpermissive temperature $\left(29^{\circ} \mathrm{C}\right)$ for 24 hours, then moved to $23^{\circ} \mathrm{C}$ until day 4 . Inka cell-blocked flies were raised at $18^{\circ} \mathrm{C}$ until eclosion, and transferred to $28^{\circ} \mathrm{C}$ until day 4 . Use of double-stranded RNA constructs for silencing of ETHR (UAS-ETHR-Sym; UAS-ETHR-IR2 line (VDRC transformant ID dna697) were

251 Institute of Mental Health, Silver Spring). GR32A-LexA, GR33A ${ }^{-/}$, and AOP-mCherry were 252 obtained from Anupama Dahanukar (University of California-Riverside). OR67D ${ }^{-/-}$was obtained 253 from Anandasankar Ray (University of California-Riverside). All other fly lines were obtained 
254 from the Bloomington Stock Center (Indiana University, Bloomington, IN): UAS-mCD8-GFP (BS 255 no. 5137), UAS-Reaper(BS no. 8524), Tubulin-Gal80's (BS no. 7017), ETH-Gal4 (BS no. 51982), 256 OR67D-Gal4 (BS no. 9998), GR32A-Gal4 (BS no. 57622). All flies used for behavior 257 experiments were backcrossed for at least five generations into the Canton-S background.

Courtship Assays

Courtship

For all behavior experiments, males were isolated prior to eclosion in culture tubes with food. Naive day 4 adult male flies were put in a $1 \mathrm{~cm}$ in diameter courtship chamber with same262 aged wild type Canton-S male or virgin female subject and observed. In the case of male-male courtship, test males were scored for stereotypical courtship behaviors and index was created as a measure of time performing those behaviors out of 10 minutes. For male female courtship, time of copulation was recorded, and percentage copulated was assessed for each minute in

266 the first ten-minute interval. Those flies that did copulate were allowed to dismount, and then

267 the male was observed and scored for courtship for the ten minutes immediately following the 268 dismount, which was recorded as post-copulation courtship index. For pre-copulation/post269 copulation comparisons, the five minutes (or less if males mated earlier) before mating was 270 compared to only the first five minutes after males and females broke copulation. Time between 271 mounting and dismounting was recorded as copulation duration. Courtship scoring was 272 performed single blind by undergraduates. 
276 each individual was tabulated and represented both as a sum and a percentage of total time

277 spent courting. Preference index was plotted as (Time courting female-Time courting male)/Total

278 time courting either subject.

\section{Immunohistochemistry}

We crossed ETHR-Gal4 transgenic flies with UAS-mCD8-GFP flies to produce progeny expressing GFP in ETHR-expressing cells for immunohistochemical staining. Day 4 adults were dissected in phosphate buffered saline (PBS) and fixed in $4 \%$ paraformaldehyde in PBS overnight at $4^{\circ} \mathrm{C}$. After washing with PBS-0.5\% Triton X-100 (PBST) five times and blocking in 3\% normal goat serum in PBST for 30 minutes at room temperature, samples were incubated with anti-NC82 (Sigma, 1:1000) anti-GFP antiserum (Invitrogen, 1:1000) dilution in PBST for 2 days at $4^{\circ} \mathrm{C}$. Tissues were washed with PBST three times, incubated with Alexa Fluor 488labeled goat anti-rabbit IgG (Invitrogen), Alexa Fluor 635-labeled Goat anti-mouse (Life 288 Technologies), $0.5 \mathrm{mg} / \mathrm{ml}$ DAPI, and 2\% NGS overnight, and washed 4 times for 10 minutes each in PBST before imaging. Immunofluorescence was recorded using a confocal microscope

290 (Leica model SP5) with FITC filter in the Institute of Integrative Genome Biology core facility at 291 UC Riverside.

\section{Extracellular tip recordings}

293 Extracellular tip-recordings from labellar taste bristles were performed as previously described 294 (Benton and Dahanukar, 2011). All tastants were dissolved in $30 \mathrm{mM}$ tricholine citrate (Sigma, 295 T0252) which functioned as the electrolyte (Wieczorek and Wolff, 1989). Recordings were 296 obtained from male flies aged 4-5 days in $25^{\circ} \mathrm{C}$. Neuronal responses were amplified and 297 digitized using an IDAC-4 data acquisition software. Visualization and manual-quantification 
298 of the action potential spikes was done by autospike software (Syntech). Number of action 299 potential spikes generated in the first 500 ms following contact artifact was used to quantify 300 the neuronal responses.

303 Males were injected with saline, 5 pmol ETH, or Faux injected using a previously described 304 procedure (Meiselman et al., 2017). In short, males were cold anesthetized and injected at 305 ZT 16:15 with 0 (faux), $50 \mathrm{nl}$ of saline, or $50 \mathrm{nl}$ of $100 \mu \mathrm{M} \mathrm{ETH}$, abdominally, from a Drummond 306 Nanoject II. 45 minutes later, each group was placed with a virgin female and scored for 307 courtship for 10 minutes.

\section{Methoprene Treatment}

309 Within 24 hours of eclosion, adult males or females were cold anesthetized and treated topically 310 on the dorsal side of the abdomen with $36 \mathrm{nl}$ of either acetone, or $0.01 \%$ methoprene dissolved 311 in acetone $(\sim 300 \mathrm{nM})$. The entire procedure took under 20 minutes, after which flies were 312 returned to their housing. 

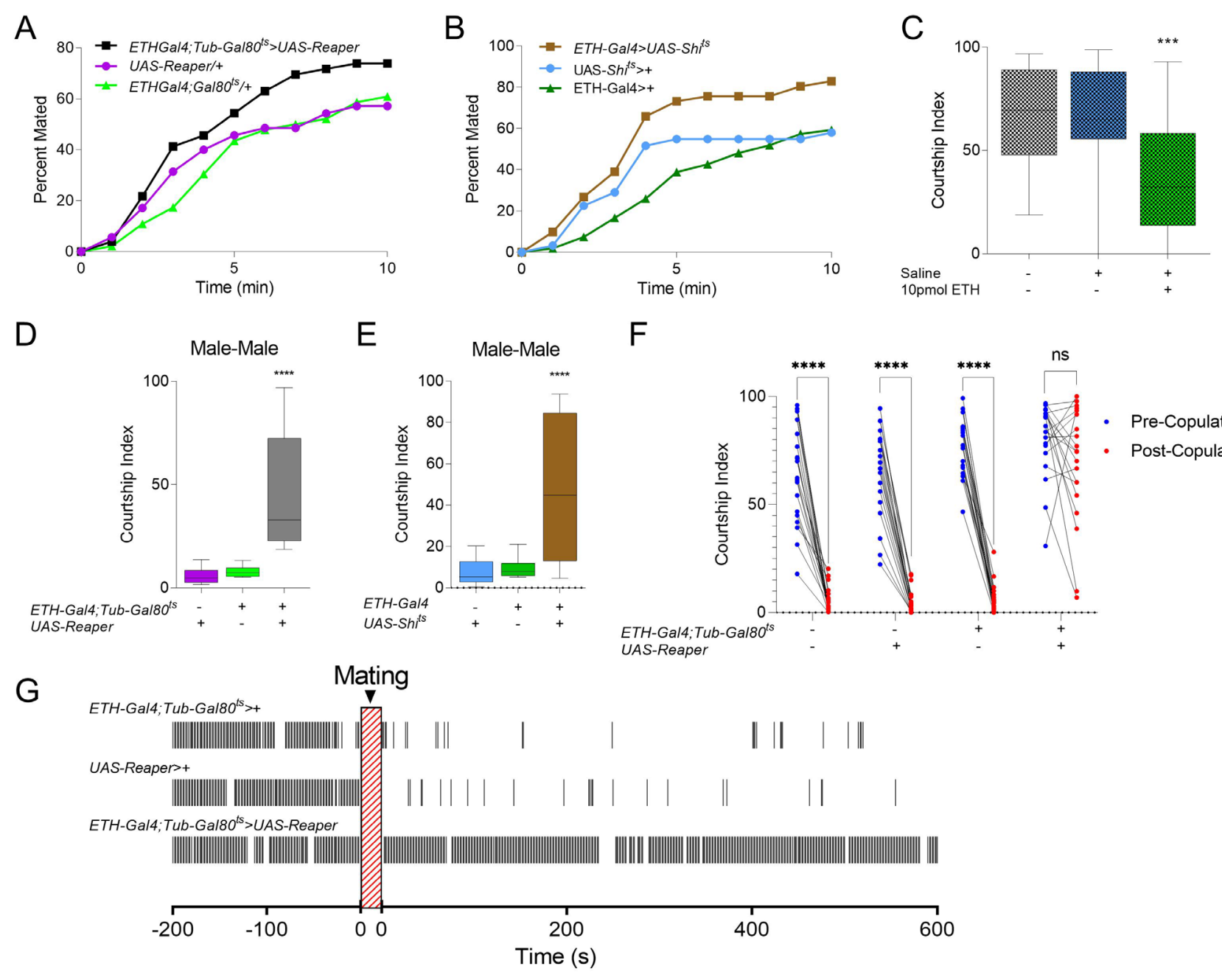

314 Figure 1. ETH-deficiency increases frequency of courtship behaviors male courtship 315 behavior toward females, males, and mated females. (A-B) Cumulative totals of males 316 successfully mating with virgin Canton-S females in ten minutes of indicated genotypes (A) Inka 317 cell-ablated, (ETH-Gal4;Tubulin-Gal80 $\left.{ }^{t s}>U A S-R e a p e r\right),(B)$ Inka cell-Blocked, (ETH-Gal4>UASShibirets $)$ and genetic controls $(n=50-60)$. (C) Male courtship index toward wt females beginning 31945 minutes after faux injection (no liquid ejected from capillary), saline injected, or injected with 3205 pmol ETH (ANOVA, $n=15)$. (C-D) Male courtship index (time spent courting over total time, 321 600s) toward Canton-S males for Inka cell-ablated (C), Inka cell blocked (D), and genetic 322 controls [One-way analysis of variance (ANOVA), $n=15-20$ ]. (E) Time courting 200 seconds 
323 before mating and 600 seconds after dismount for an example Inka cell-ablated male and genetic 324 controls. Black bars represent seconds performing courtship behavior. (F) Comparison of 325 courtship index before (Blue dots) and 300 seconds after (Red dots) successful mating for 326 Canton-S, Inka cell-ablated males and controls (bootstrap, $\mathrm{n}=20$ ) ns $p>.05 ;{ }^{* *} p<.01 ;{ }^{* * *} p<$ $327.001 ;{ }^{* * *} p<.0001$. 


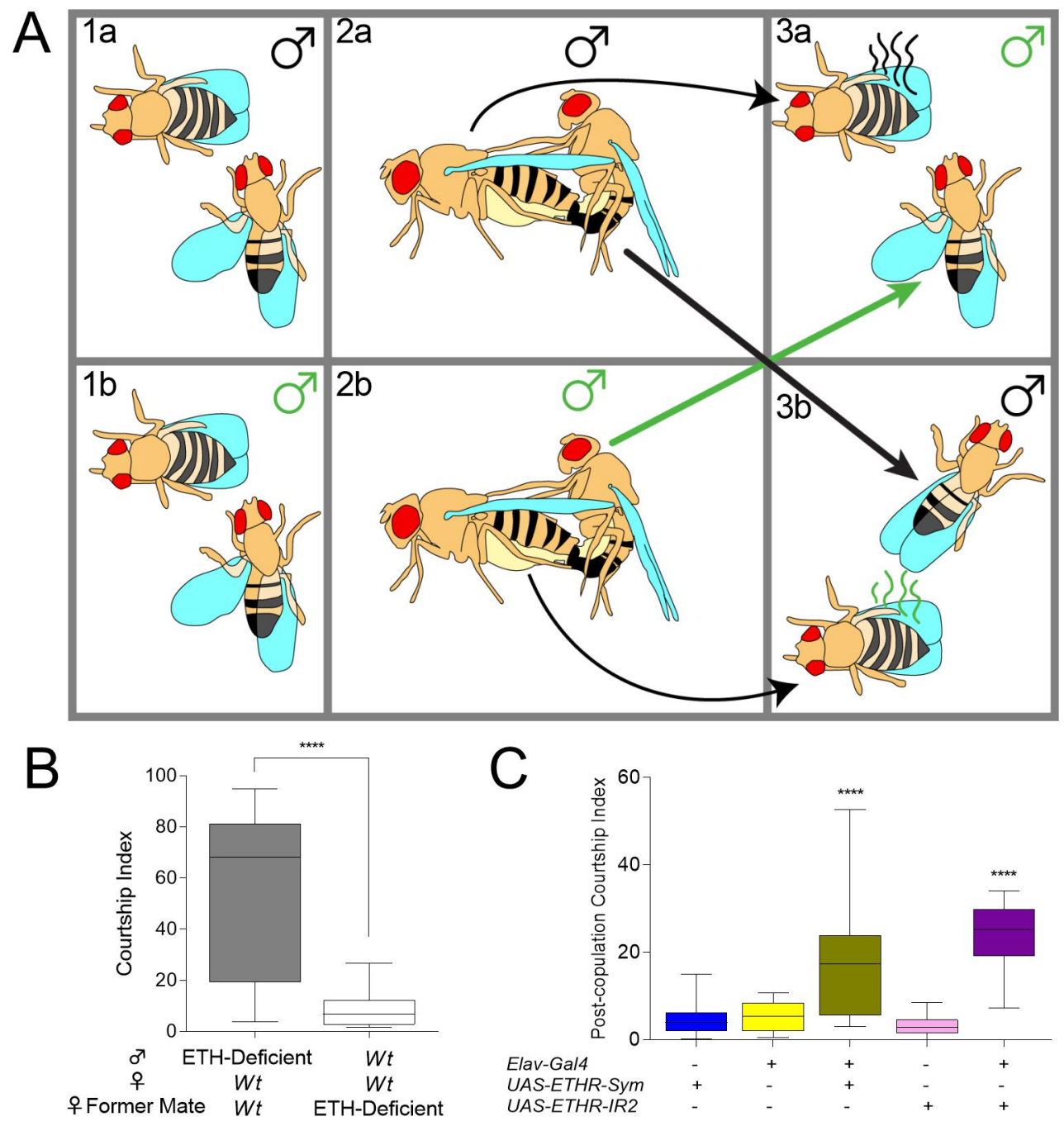

Figure 2. ETH inhibits courtship via perception. (A) Diagram for Mate and Switch experiment. Wt and ETH-deficient males court and mate wt female counterparts (1a-2a, 1b-2b, respectively). After completion of mating, ETH-deficient males are placed with females mated to wt male counterparts (3a), and wt males are aspirated and placed with females recently mated to ETHdeficient males from opposite panel (3b). (B) Courtship index after mate and switch for ETHDeficient and wt males after mating and switching (Mann-whitney $U, n=15$ ). (C) Male postcopulation courtship index for flies with ETHR knocked down pan-neuronally (Elav-Gal4>UASETHR-RNAi) and genetic controls (ANOVA, $\mathrm{n}=20-21)$. ns $p>.05 ;{ }^{* * *} p<.0001$ 


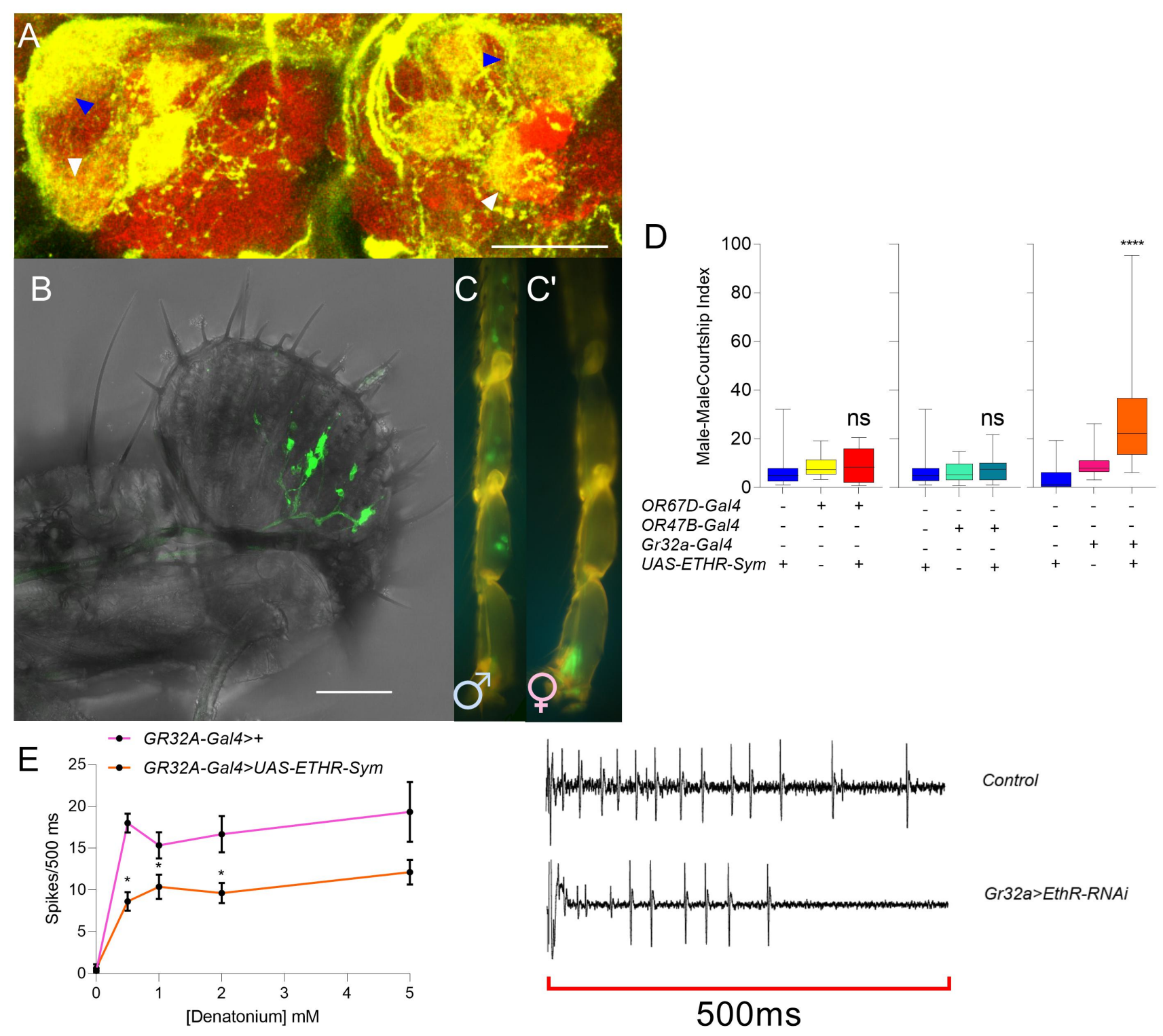

343 Figure 3. ETHR knockdown reduces sensitivity to ligands in pheromone-sensing 344 neurons. (A) Antennal lobes of ETHR-trojan-Gal4>UAS-mCD8-GFP males with arrowheads 345 indicating DA1 glomerulus (targeted by OR67D+ neurons, blue) and VA1l/m glomerulus 346 (targeted by OR47B, white)(scale bar $50 \mu \mathrm{m})$. (B-C) ETHR-trojan-Gal4>UAS-mCD8-GFP 347 expression in male labellum (D), and in prothoracic tarsi (E)(scale bar $50 \mu \mathrm{m})$. Sensory hairs are 348 labeled in male tarsi (C), but not females (C'). (D) Male-male courtship index of males with ETHR 349 silenced in pheromone sensory neurons OR67D, OR47B, and GR32A with genetic controls 
350 (ANOVA, $n=20-25$ ). (E) Extracellular recordings from $S 6$ taste hairs of GR32A-Gal4>UAS351 ETHR-Sym and GR32A-Gal4/+ males to $0,0.5,1,2$, and $5 \mathrm{mM}$ denatonium for $500 \mathrm{~ms}$ after 352 contact (t-test, $\mathrm{n}=6-8)$. (F) Example spike for counts. ns $p>.05 ;{ }^{*} p<.05 ;{ }^{* * *} p<.0001$. 353 
A
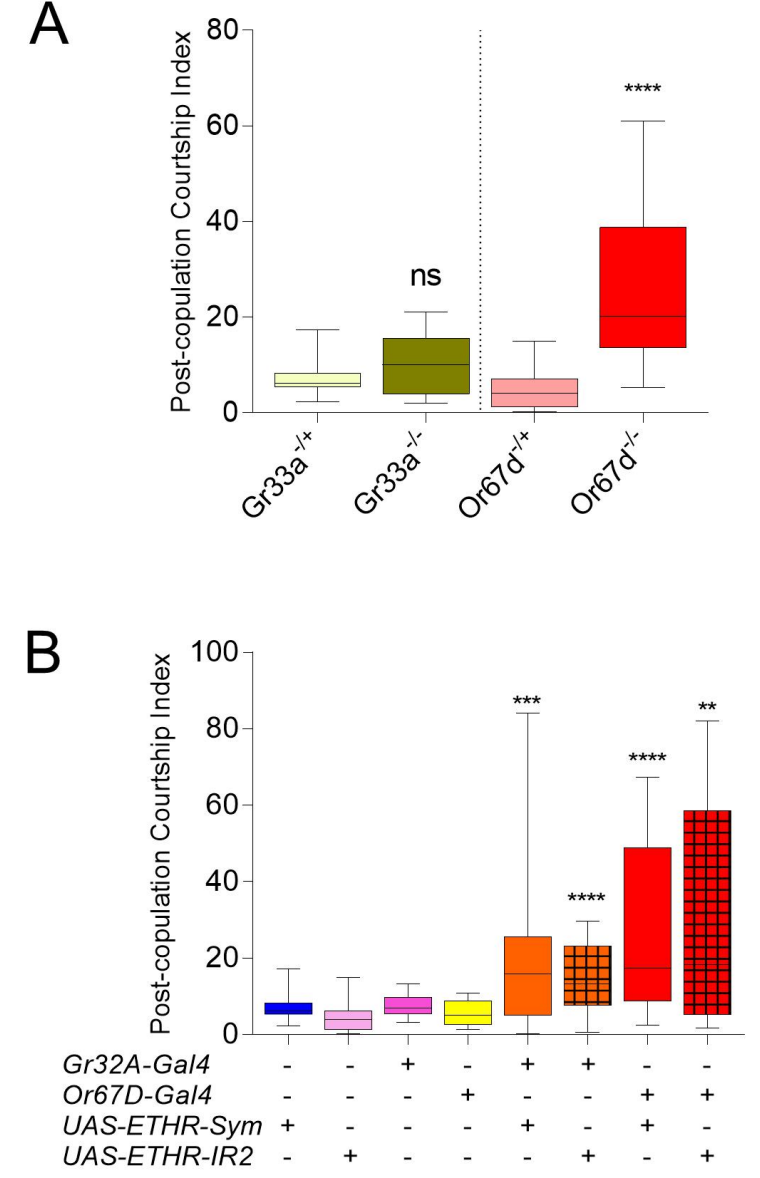

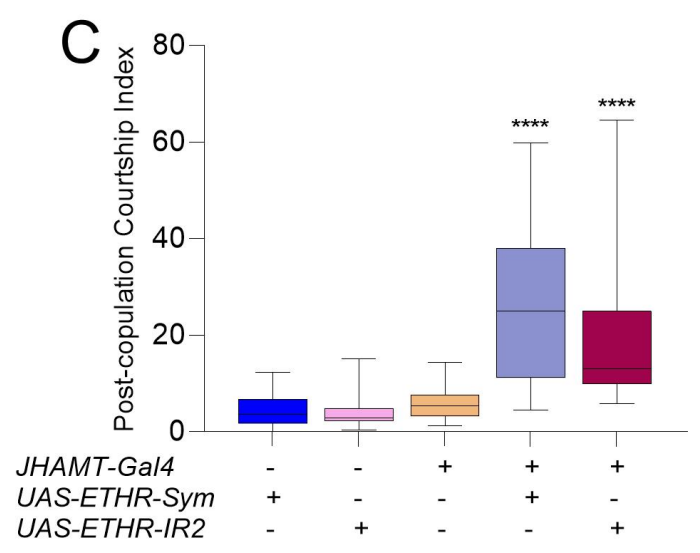

Inka Cell-ablated Males

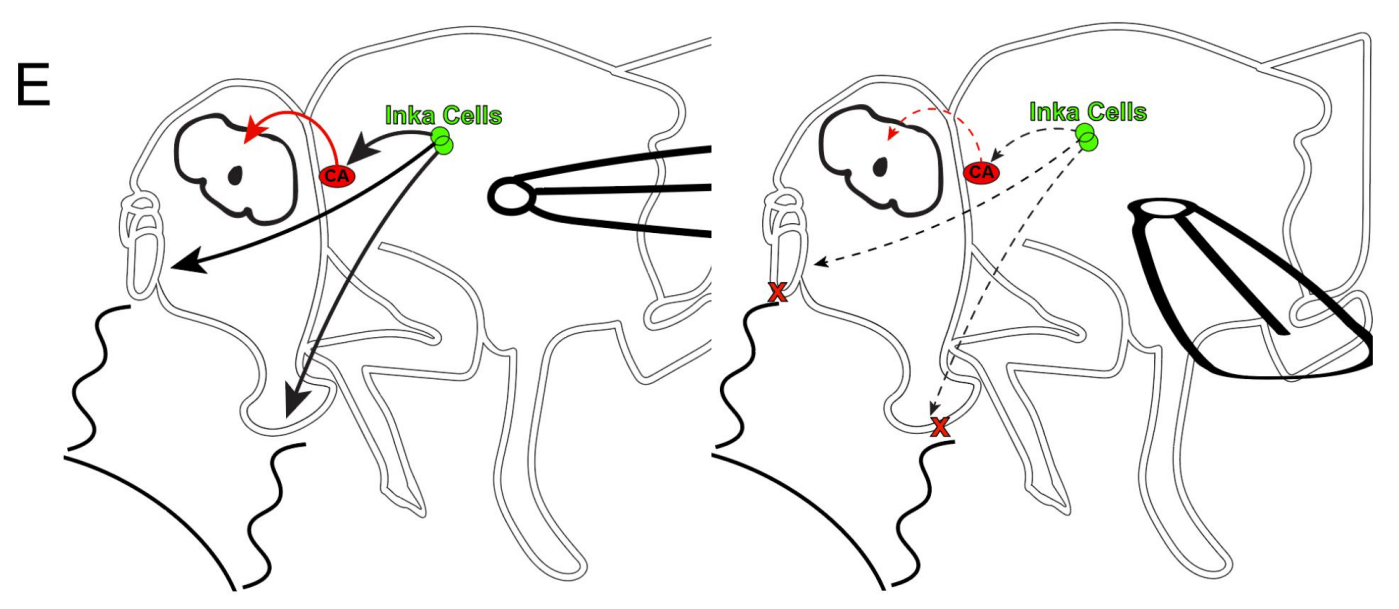
neurons, and JH levels. (A) Post-copulation courtship for GR33A ${ }^{-/-}$and OR67D ${ }^{-/-}$and 358 heterozygous controls (Mann-Whitney $U, n=10-18$ ). (B) Post-copulation courtship index for GR32A-Gal4>UAS-ETHR-RNAi, OR67D-Gal4>UAS-ETHR-RNAi and genetic controls 
(ANOVA, n=10-25). (C) Post-copulation Courtship for JHAMT-Gal4>UAS-ETHR-RNAi flies and

361 controls. Pre-copulation and post-copulation courtship for Inka cell-ablated (ETH-Gal4;Tub-

362 Gal80ts $>$ UAS-Reaper) males treated with either acetone alone or acetone with the Juvenile

363 Hormone Analog, methoprene $(n=10-12)$. (E) Model for the role of ETH in modulation of 364 courtship. In normal conditions (Left), Inka cells (green) release ETH, stimulating Juvenile 365 Hormone synthesis via the Corpus Allatum (Red) and subsequent inhibition of P1 circuitry. 366 Simultaneously, ETH promotes sensitivity to aversive pheromones in peripheral sensory 367 neurons. When ETH is low, the CA and aversive pheromone-sensing neurons are less active, disinhibiting courtship. ns $p>0.05 ;{ }^{* *} p<.01 ;{ }^{* * *} p<0.001 ;{ }^{* * * *} p<.0001$.
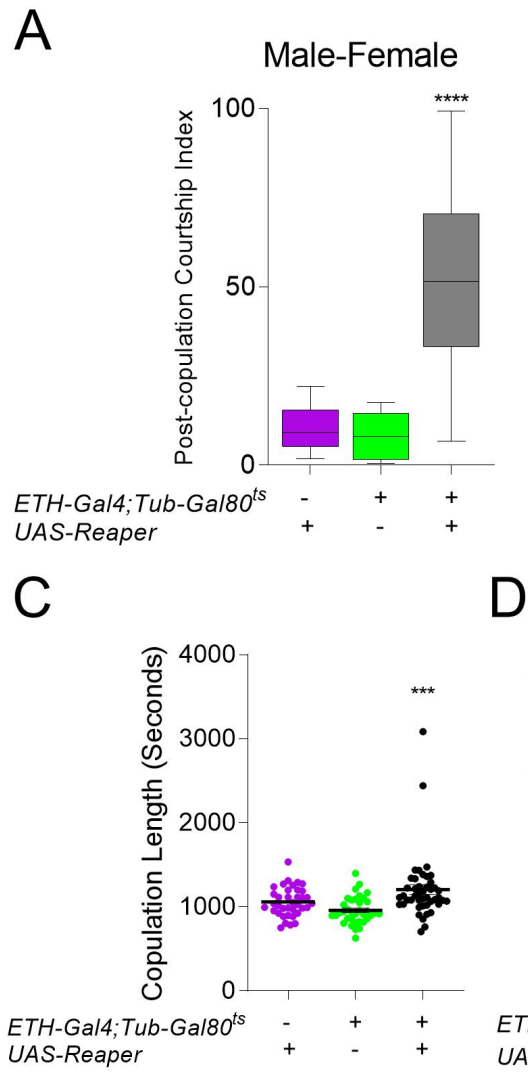

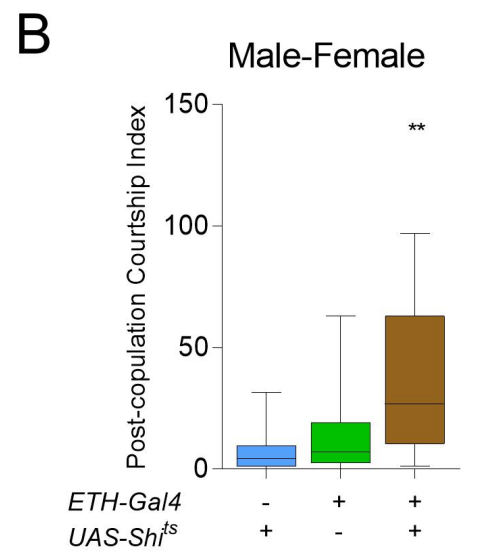

D

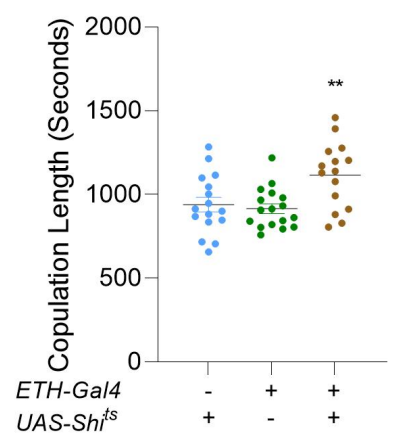

ETH-Gal4;Tub-Gal80 UAS-ECR-RNAi

$\mathrm{E}$

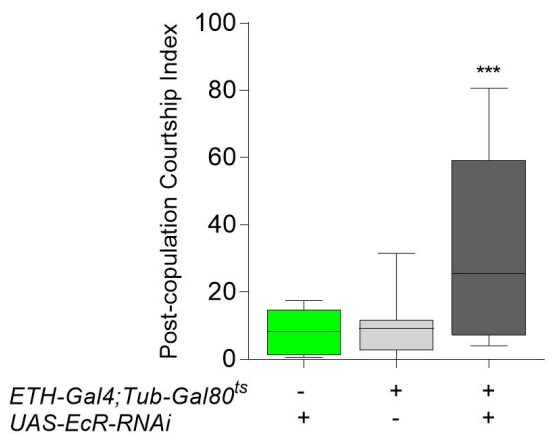


372 courtship index (time spent courting over total time starting at dismounting, 600s) toward a wt

373 females for Inka cell-ablated (C), Inka cell blocked (D), and genetic controls (ANOVA, n=20-30).

374 (C-D) Copulation duration (seconds from mounting to dismount) for Inka cell-ablated (ETH375 Gal4;Tub-Gal80's $>$ UAS-Reaper $)(\mathrm{A}, \mathrm{n}=30-40)$ and Inka cell-blocked $\left(E T H-G a l 4>U A S-S h i^{i s}\right)(\mathrm{B}$, $376 \mathrm{n}=15-20$ ). (E) Post-mating courtship index (time spent courting over total time starting at 377 dismounting, 600s) toward a wt females with EcR-silenced in Inka cells and genetic controls 378 (ANOVA, $\mathrm{n}=10-20) . \mathrm{ns} \mathrm{p}>0.05 ;{ }^{* *} p<.01 ;{ }^{* * *} p<0.001 ;{ }^{* * * *} p<.0001$

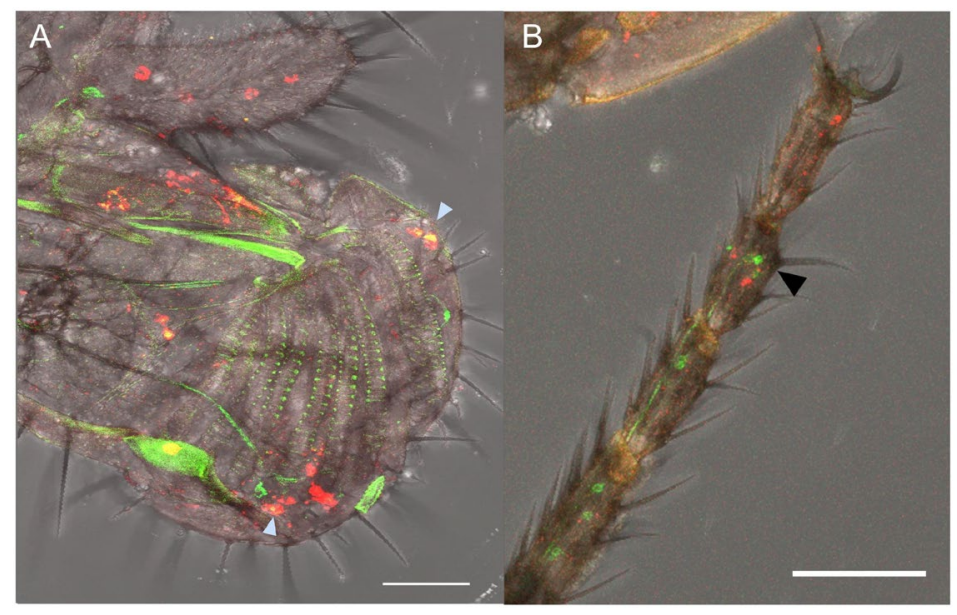

382 Figure S2. Male expression pattern of ETHR-Gal4 (A-B) UAS-mCD8;ETHR-Gal4/Aop383 mCherry;GR32A-LexA overlapping expression in labella (A, blue arrowheads) non-overlapping expression in tarsi (B, black arrowhead). 


\section{References}

Benton, R., and Dahanukar, A. (2011). Electrophysiological recording from Drosophila taste sensilla. Cold Spring Harb Protoc 2011, 839-850.

Bontonou, G., and Wicker-Thomas, C. (2014). Sexual Communication in the Drosophila Genus. Insects 5, 439-458. Chapman, T., Liddle, L.F., Kalb, J.M., Wolfner, M.F., and Partridge, L. (1995). Cost of mating in Drosophila melanogaster females is mediated by male accessory gland products. Nature 373, 241-244. Chenoweth, P.J. (1981). Libido and Mating-Behavior in Bulls, Boars and Rams - a Review. Theriogenology 16, 155177.

Cho, K.H., Daubnerova, I., Park, Y., Zitnan, D., and Adams, M.E. (2014). Secretory competence in a gateway endocrine cell conferred by the nuclear receptor betaFTZ-F1 enables stage-specific ecdysone responses throughout development in Drosophila. Dev Biol 385, 253-262.

Clowney, E.J., Iguchi, S., Bussell, J.J., Scheer, E., and Ruta, V. (2015). Multimodal Chemosensory Circuits Controlling Male Courtship in Drosophila. Neuron 87, 1036-1049.

Demir, E., and Dickson, B.J. (2005). fruitless splicing specifies male courtship behavior in Drosophila. Cell 121, 785794.

Deshpande, S.A., Meiselman, M., Hice, R.H., Arensburger, P., Rivera-Perez, C., Kim, D.H., Croft, R.L., Noriega, F.G., and Adams, M.E. (2019). Ecdysis triggering hormone receptors regulate male courtship behavior via antennal lobe interneurons in Drosophila. Gen Comp Endocrinol 278, 79-88.

Ejima, A., Smith, B.P.C., Lucas, C., Van Naters, W.V., Miller, C.J., Carlson, J.R., Levine, J.D., and Griffith, L.C. (2007). Generalization of courtship learning in Drosophila is mediated by cis-vaccenyl acetate. Current Biology 17, 599605.

Gillott, C. (2003). Male accessory gland secretions: Modulators of female reproductive physiology and behavior. Annual Review of Entomology 48, 163-184.

Gonzalez-Bellido, P.T., Wardill, T.J., Kostyleva, R., Meinertzhagen, I.A., and Juusola, M. (2009). Overexpressing Temperature-Sensitive Dynamin Decelerates Phototransduction and Bundles Microtubules in Drosophila Photoreceptors. Journal of Neuroscience 29, 14199-14210.

Ishimoto, H., Sakai, T., and Kitamoto, T. (2009). Ecdysone signaling regulates the formation of long-term courtship memory in adult Drosophila melanogaster. Proc Natl Acad Sci U S A 106, 6381-6386.

Jallon, J.M. (1984). A Few Chemical Words Exchanged by Drosophila during Courtship and Mating. Behav Genet 14, 441-478.

Kim, Y.J., Zitnan, D., Galizia, C.G., Cho, K.H., and Adams, M.E. (2006). A command chemical triggers an innate behavior by sequential activation of multiple peptidergic ensembles. Current Biology 16, 1395-1407.

Kimura, K., Hachiya, T., Koganezawa, M., Tazawa, T., and Yamamoto, D. (2008). Fruitless and Doublesex Coordinate to Generate Male-Specific Neurons that Can Initiate Courtship (vol 59, pg 759, 2008). Neuron 60, 390-391.

Kurtovic, A., Widmer, A., and Dickson, B.J. (2007). A single class of olfactory neurons mediates behavioural responses to a Drosophila sex pheromone. Nature 446, 542-546.

Lacaille, F., Hiroi, M., Twele, R., Inoshita, T., Umemoto, D., Maniere, G., Marion-Poll, F., Ozaki, M., Francke, W., Cobb, M., et al. (2007). An Inhibitory Sex Pheromone Tastes Bitter for Drosophila Males. Plos One 2.

Laturney, M., and Billeter, J.C. (2016). Drosophila melanogaster females restore their attractiveness after mating by removing male anti-aphrodisiac pheromones. Nature Communications 7.

Lee, S.S., and Adams, M.E. (2021). Regulation of Drosophila Long-Term Courtship Memory by Ecdysis Triggering Hormone. Front Neurosci-Switz 15.

Lee, S.S., Ding, Y.K., Karapetians, N., Rivera-Perez, C., Noriega, F.G., and Adams, M.E. (2017). Hormonal Signaling Cascade during an Early-Adult Critical Period Required for Courtship Memory Retention in Drosophila. Current Biology 27, 2798-+.

Leinwand, S.G., and Scott, K. (2021). Juvenile hormone drives the maturation of spontaneous mushroom body neural activity and learned behavior. Neuron 109, 1836-+. 
Lenzi, A., Lombardo, F., Salacone, P., Gandini, L., and Jannini, E.A. (2003). Stress, sexual dysfunctions, and male infertility. J Endocrinol Invest 26, 72-76.

Liang, L., Li, Y.L., Potter, C.J., Yizhar, O., Deisseroth, K., Tsien, R.W., and Luo, L.Q. (2013). GABAergic Projection Neurons Route Selective Olfactory Inputs to Specific Higher-Order Neurons. Neuron 79, 917-931.

Lin, H.H., Cao, D.S., Sethi, S., Zeng, Z., Chin, J.S.R., Chakraborty, T.S., Shepherd, A.K., Nguyen, C.A., Yew, J.Y., Su, C.Y., et al. (2016). Hormonal Modulation of Pheromone Detection Enhances Male Courtship Success. Neuron 90, $1272-1285$.

Liu, H.F., and Kubli, E. (2003). Sex-peptide is the molecular basis of the sperm effect in Drosophila melanogaster. P Natl Acad Sci USA 100, 9929-9933.

Liu, W.W., Liang, X.H., Gong, J.X., Yang, Z., Zhang, Y.H., Zhang, J.X., and Rao, Y. (2011). Social regulation of aggression by pheromonal activation of Or65a olfactory neurons in Drosophila. Nature Neuroscience 14, 896U119.

Marai, I.F.M., Habeeb, A.A.M., and Gad, A.E. (2002). Rabbits' productive, reproductive and physiological performance traits as affected by heat stress: a review. Livest Prod Sci 78, 71-90.

Markow, T.A., Quaid, M., and Kerr, S. (1978). Male Mating Experience and Competitive Courtship Success in Drosophila-Melanogaster. Nature 276, 821-822.

Meiselman, M., Lee, S.S., Tran, R.T., Dai, H., Ding, Y., Rivera-Perez, C., Wijesekera, T.P., Dauwalder, B., Noriega, F.G., and Adams, M.E. (2017). Endocrine network essential for reproductive success in Drosophila melanogaster. Proc Natl Acad Sci U S A 114, E3849-E3858.

Meiselman, M.R., Kingan, T.G., and Adams, M.E. (2018). Stress-induced reproductive arrest in Drosophila occurs through ETH deficiency-mediated suppression of oogenesis and ovulation. BMC Biol 16, 18.

Moon, S.J., Lee, Y., Jiao, Y., and Montell, C. (2009). A Drosophila Gustatory Receptor Essential for Aversive Taste and Inhibiting Male-to-Male Courtship. Current Biology 19, 1623-1627.

Moshitzky, P., Fleischmann, I., Chaimov, N., Saudan, P., Klauser, S., Kubli, E., and Applebaum, S.W. (1996). Sexpeptide activates juvenile hormone biosynthesis in the Drosophila melanogaster corpus allatum. Arch Insect Biochem 32, 363-374.

Pan, Y.F., Meissner, G.W., and Baker, B.S. (2012). Joint control of Drosophila male courtship behavior by motion cues and activation of male-specific P1 neurons. P Natl Acad Sci USA 109, 10065-10070.

Park, Y., Kim, Y.J., Dupriez, V., and Adams, M.E. (2003). Two subtypes of ecdysis-triggering hormone receptor in Drosophila melanogaster. Journal of Biological Chemistry 278, 17710-17715.

Ribeiro, I.M.A., Drews, M., Bahl, A., Machacek, C., Borst, A., and Dickson, B.J. (2018). Visual Projection Neurons Mediating Directed Courtship in Drosophila. Cell 174, 607-+.

Rings, A., and Goodwin, S.F. (2019). To court or not to court - a multimodal sensory decision in Drosophila males. Curr Opin Insect Sci 35, 48-53.

Sugime, Y., Watanabe, D., Yasuno, Y., Shinada, T., Miura, T., and Tanaka, N.K. (2017). Upregulation of Juvenile Hormone Titers in Female Drosophila melanogaster Through Mating Experiences and Host Food Occupied by Eggs and Larvae. Zool Sci 34, 52-57.

Teal, P.E.A., Gomez-Simuta, Y., and Proveaux, A.T. (2000). Mating experience and juvenile hormone enhance sexual signaling and mating in male Caribbean fruit flies. P Natl Acad Sci USA 97, 3708-3712.

van Naters, W.V.G., and Carlson, J.R. (2007). Receptors and neurons for fly odors in Drosophila. Current Biology $17,606-612$.

Vandermeer, R.K., Obin, M.S., Zawistowski, S., Sheehan, K.B., and Richmond, R.C. (1986). A Reevaluation of the Role of Cis-Vaccenyl Acetate, Cis-Vaccenol and Esterase- 6 in the Regulation of Mated Female Sexual Attractiveness in Drosophila-Melanogaster. Journal of Insect Physiology 32, 681-686.

Wang, L., Han, X., Mehren, J., Hiroi, M., Billeter, J.C., Miyamoto, T., Amrein, H., Levine, J.D., and Anderson, D.J. (2011). Hierarchical chemosensory regulation of male-male social interactions in Drosophila. Nat Neurosci 14, 757-762.

White, K., Tahaoglu, E., and Steller, H. (1996). Cell killing by the Drosophila gene reaper. Science 271, 805-807. 
484 Wieczorek, H., and Wolff, G. (1989). The Labellar Sugar Receptor of Drosophila. J Comp Physiol A 164, 825-834. 485 Wijesekera, T.P., Saurabh, S., and Dauwalder, B. (2016). Juvenile Hormone Is Required in Adult Males for 486 Drosophila Courtship. Plos One 11.

487 Wu, S.F., Guo, C., Zhao, H., Sun, M.S., Chen, J., Han, C.H., Peng, Q.L., Qiao, H.H., Peng, P., Liu, Y., et al. (2019). 488 Drosulfakinin signaling in fruitless circuitry antagonizes P1 neurons to regulate sexual arousal in Drosophila. 489 Nature Communications 10.

490 Yamamoto, K., Chadarevian, A., and Pellegrini, M. (1988). Juvenile-Hormone Action Mediated in Male Accessory491 Glands of Drosophila by Calcium and Kinase-C. Science 239, 916-919.

492 Zhang, S.X., Glantz, E.H., Miner, L.E., Rogulja, D., and Crickmore, M.A. (2021). Hormonal control of motivational 493 circuitry orchestrates the transition to sexuality in Drosophila. Sci Adv 7.

494 Zhang, S.X., Rogulja, D., and Crickmore, M.A. (2016). Dopaminergic Circuitry Underlying Mating Drive. Neuron 91, 495 168-181.

496 Zhang, S.X., Rogulja, D., and Crickmore, M.A. (2019). Recurrent Circuitry Sustains Drosophila Courtship Drive While 497 Priming Itself for Satiety. Curr Biol 29, 3216-3228 e3219. 